\title{
Extended mid-infrared emission from VV 114: Probing the birth of a ULIRG ^
}

\author{
E. Le Floc' $h^{1}$, V. Charmandaris ${ }^{2}$, O. Laurent ${ }^{1,3}$, I. F. Mirabel ${ }^{1,4}$, P. Gallais ${ }^{1}$, M. Sauvage ${ }^{1}$, \\ L. Vigroux ${ }^{1}$, and C. Cesarsky ${ }^{5}$ \\ ${ }^{1}$ CEA/DSM/DAPNIA Service d'Astrophysique, 91191 Gif-sur-Yvette, France \\ 2 Cornell University, Astronomy Department, Ithaca, NY 14853, USA \\ 3 Max Planck Institut für extraterrestrische Physik, Postfach 1312, 85741 Garching, Germany \\ ${ }^{4}$ Instituto de Astronomía y Física del Espacio, cc 67, suc 28. 1428 Buenos Aires, Argentina \\ 5 European Southern Observatory, Karl-Schwarzschild-Str, 85748 Garching bei München, Germany
}

Received 4 March 2002 / Accepted 22 May 2002

\begin{abstract}
We present our 5-16 $\mu \mathrm{m}$ spectro-imaging observations of VV 114, an infrared luminous early-stage merger of two galaxies VV 114E and VV 114W, taken with the ISOCAM camera on-board the Infrared Space Observatory. We find that only $40 \%$ of the mid-infrared (MIR) flux is associated with a compact nuclear region of VV 114E, while the rest of the emission originates from a rather diffuse component extended over several kpc in the regions between VV 114E and VV 114W. This is in stark contrast with the very compact MIR starbursts usually seen in luminous and ultraluminous infrared galaxies. A secondary peak of MIR emission is associated with an extra-nuclear star forming region of VV 114W which displays the largest $\mathrm{H} \alpha$ equivalent width in the whole system. Comparing our data with the distribution of the molecular gas and cold dust, as well as with radio observations, it becomes evident that the conversion of molecular gas into stars can be triggered over large areas at the very first stages of an interaction. This extended star formation along with the extreme nuclear starburst observed in VV 114E can easily lead to the heating of dust grains found in the tidally disturbed disks of the progenitor galaxies and subsequently traced via their MIR emission. The presence of a very strong continuum at the 5-6.5 $\mu \mathrm{m}$ range in the spectrum of VV 114E indicates that an enshrouded active galactic nucleus (AGN) may contribute to $40 \%$ of its MIR flux. We finally note that the relative variations in the UV to radio spectral properties between VV 114E and VV 114W provide evidence that the extinctioncorrected star formation rate of similar objects at high $\mathrm{z}$, such as those detected in optical deep surveys, cannot be accurately derived from their rest-frame UV properties.
\end{abstract}

Key words. galaxies: active - galaxies: starburst - galaxies: ISM - galaxies: individual: ARP 236 galaxies: individual: VV 114 - infrared: galaxies

\section{Introduction}

The first all-sky survey at far-infrared (FIR) wavelengths performed by IRAS in 1983 led to the discovery of a large number of galaxies with $L_{\mathrm{IR}} \geq 10^{11} L_{\odot}$, the so-called luminous infrared galaxies (see Rieke \& Low 1972; Soifer et al. 1989). Ever since, extensive work has been devoted to better understand the nature of these objects that emit the bulk of their energy in the $8-1000 \mu \mathrm{m}$ domain, and it is now widely accepted that the brightest of these sources are formed during the collisions of massive gas-rich late type galaxies (see Sanders \& Mirabel 1996, and references therein). In such galactic

Send offprint requests to: E. Le Floc'h, e-mail: elefloch@cea.fr

* Based on observations with the ISO satellite, an ESA project with instruments funded by ESA Member States (especially the PI countries: France, Germany, the Netherlands and the United Kingdom) and with the participation of ISAS and NASA. encounters, as the tidal interactions strip angular momentum from the interstellar matter, large concentrations of molecular gas are indeed driven by viscous accretion to the few central $100 \mathrm{pc}$ of the merging galaxies (Solomon et al. 1997; Combes 2001), feeding at high rates powerful circumnuclear starbursts and/or supermassive black holes (Sanders et al. 1988). The huge amounts of dust accumulated into the central regions lead to a significant absorption of the UV and optical radiation which in turn is thermally reprocessed at longer wavelengths, and is thus responsible for the luminous phase in the infrared. Several key problems though, such as the time evolution and duration of the infrared burst during the merger event, as well as the identification of the dominant sources heating the dust (stars, AGN) are still not fully answered. Observations of colliding galaxies at different merging stages are therefore of a prime importance to better understand how the AGN and starburst phenomena are related (see Mihos \& Hernquist 1996). 
The interest in the study of these objects was further increased over the past few years when it became evident that they also played a critical role in galaxy evolution over cosmological look-back times. Luminous infrared galaxies of the local Universe are indeed excellent analogs to the high-redshift dusty objects recently discovered in deep mid-infrared and submillimeter surveys (e.g. Elbaz et al. 1999; Hughes et al. 1998). They are thus considered as the low- $\mathrm{z}$ counterparts of a population of dust-enshrouded sources that were formed at an epoch when interactions and mergers among galaxies were much more frequent than today (Le Fèvre et al. 2000). Consequently, investigating the properties of colliding galaxies and the formation mechanisms of sources luminous in the infrared (IR) at low redshift can provide valuable insights on the evolution of galaxies in the distant Universe.

VV 114 (= Arp $236=$ IC 1623), located at a distance of $80 \mathrm{Mpc}^{1}\left(1^{\prime \prime}=390 \mathrm{pc}\right)$, is one of such interacting systems undergoing vigorous starburst activity. Its infrared luminosity is $L_{\mathrm{IR}}=10^{11.6} L_{\odot}$ (Soifer et al. 1987), making it a $\mathrm{LIRG}^{2}$ and one of the brightest objects of the IRAS Bright Galaxy Sample. It appears to be an early-stage merger of two galaxies (see Fig. 1) that are aligned east-west with a projected nuclear separation of $\sim 6 \mathrm{kpc}$ and therefore referred in the literature as VV 114E and VV 114W (Knop et al. 1994). The global properties of the system are summarized in Table 1. At optical wavelengths, VV 114 shows a highly disturbed morphology with very faint tidal tails extending over $25 \mathrm{kpc}$ from the center (Arp 1966). The western component, VV $114 \mathrm{~W}$, is more extended than the eastern one, and dominates the emission in the visible. Three peaks of intensity are clearly visible in the $V$ band and their optical spectrum indicates an emission typical of $\mathrm{H}_{\text {II }}$ regions with an average visual extinction $A_{\mathrm{v}}=1.7 \mathrm{mag}$ (Knop et al. 1994). While the brightest peak to the North (knot 1) probably coincides with the dynamical center of VV 114W (Doyon et al. 1995), the maximum of star formation traced by the $\mathrm{H} \alpha$ line is displaced and it occurs in the secondary peak (knot 2) which has an $\mathrm{H} \alpha$ equivalent width six times larger than knot 1 , and is located 5" to the southeast in the overlap region of the two galactic disks (Knop et al. 1994).

The near-infrared (NIR) morphology and spectral properties of VV 114 present a remarkable contrast with what is observed in the visible. Medium resolution NIR spectra of both components display strong recombination lines of hydrogen and helium as well as deep CO bands at $2.3 \mu \mathrm{m}$, indicating vigorous and widespread star formation in both galaxies (Doyon et al. 1995). However, whereas the JHK colors of VV $114 \mathrm{~W}$ are still consistent with the presence of numerous $\mathrm{H}$ II regions, the continuum emission from VV 114E which is nearly invisible at $0.44 \mu \mathrm{m}$ ( $B$ band), becomes brighter as the wavelength increases. Its red colors indicate large concentrations of dust ( $A_{\mathrm{v}} \sim 4 \mathrm{mag}$, Doyon et al. 1995) obscuring a luminous source

\footnotetext{
${ }^{1}$ Throughout the paper, we adopt $H_{0}=75 \mathrm{~km} \mathrm{~s}^{-1} \mathrm{Mpc}^{-1}$ and $q_{\mathrm{o}}=$ 0.5 to facilitate comparison with earlier work.

${ }^{2}$ We define LIRGs as galaxies with $10^{11} L_{\odot} \leq L_{\mathrm{IR}} \leq 10^{12} L_{\odot}$, and ULIRGs as ultraluminous sources with $L_{\mathrm{IR}} \geq 10^{12} L_{\odot}$. We keep the more general expression of "luminous infrared galaxies" for those with $L_{\mathrm{IR}} \geq 10^{11} L_{\odot}$.
}

which is unveiled at $2 \mu \mathrm{m}$ and dominates the whole emission of the system in the near-infrared (Knop et al. 1994). High resolution $K$-band images of this eastern core taken with HST/NICMOS reveal a double-nucleus (see Fig. 2 in Scoville et al. 2000) with the two near-infrared sources separated by $1.6^{\prime \prime}(\sim 630 \mathrm{pc})$. The NICMOS data clearly show numerous compact $\mathrm{H}$ II regions and star clusters distributed along a spiral arm in the overlap of the merger, which suggests an enhancement of the starburst activity in this area (Scoville et al. 2000), in agreement with the $\mathrm{H} \alpha$ observations (Knop et al. 1994).

The presence of a powerful starburst in VV 114 is also suggested by its huge amount of molecular gas $\left(5.1 \times 10^{10} M_{\odot}\right.$, see Yun et al. 1994), which makes this merger one of the most gas-rich systems in the local Universe. The CO emission is distributed along a bar-shaped structure joining VV 114E and VV $114 \mathrm{~W}$, with a peak of intensity centered between the two merging galaxies and its irregular kinematics suggest that the gas has not yet settled (Yun et al. 1994). Submillimeter observations performed with SCUBA on the JCMT have also detected an extended dust emission over $30^{\prime \prime}(12 \mathrm{kpc})$, which correlates well with the spatial distribution of the $\mathrm{CO}$ emission (Frayer et al. 1999) and peaks just 5" southwest of VV 114E. The flux observed at 450 and $850 \mu \mathrm{m}$ is in excess of that expected from the IRAS data and suggests the presence of a cool $(T \sim 20-25 \mathrm{~K})$ and massive $\left(10^{8} M_{\odot}\right)$ dust component (Frayer et al. 1999). Finally, VV 114 also exhibits a strong radio continuum emission, with a ratio of FIR to radio radiation typical of that observed in the IRAS Bright Galaxy Sample (Condon et al. 1990). Its $1.49 \mathrm{GHz}$ emission globally follows the distribution seen in the visible and it extends along the overlap region between the east and western components. A local maximum of the radio emission is seen to coincide with the brightest $\mathrm{H} \alpha$ emission in the overlap region, while VV 114E displays a double-peak radio morphology similar to that observed at $2.2 \mu \mathrm{m}$ (Condon et al. 1990, 1991). The spatial coincidence of these two sources at NIR and radio wavelengths combined with the similarity of the two nuclei in terms of the NIR/radio flux ratio and the CO kinematics suggest that VV 114E itself may have experienced an earlier merger.

Since observations in the mid-infrared (MIR) are less affected by absorption than in the visible $\left(A_{15 \mu \mathrm{m}} \sim A_{V} / 70\right.$, see Mathis 1990), they provide a powerful tool to probe deeper into the dust-enshrouded regions of galaxies, and allow one to trace the reprocessed emission of dust heated by UV radiation. Recent high resolution MIR images of VV 114 obtained at Keck by Soifer et al. (2001) led to a significant improvement in our understanding of that system - discussed in detail in the following sections - despite the fact that ground-based observations generally suffer from the low transparency of the atmosphere at these wavelengths. We thus used the high sensitivity and the good spatial and spectral resolution of the ISOCAM camera to gain a new insight in the mid-infrared properties of VV 114. After describing the ISO observations and data reduction in Sect. 2, we present our analysis on the distribution of the MIR emission in Sect. 3.1 and the spectral properties of VV 114 in Sect. 3.2. We discuss our results in Sect. 4 and summarize our conclusions in Sect. 5. 
Table 1. Global properties of VV 114.

\begin{tabular}{lr}
\hline \hline Parameter & Value \\
\hline$\alpha(\mathrm{J} 2000.0) \mathrm{VV} 114 \mathrm{E}^{a}$ & $01^{\mathrm{h}} 07^{\mathrm{m}} 47^{\$} .5$ \\
$\delta(\mathrm{J} 2000.0) \mathrm{VV} 114 \mathrm{E}^{a}$ & $-17^{\circ} 30^{\prime} 25^{\prime \prime}$ \\
Distance $^{b}$ & $80 \mathrm{Mpc}$ \\
$L_{\mathrm{IR}}{ }^{c}$ & $10^{11.6} L_{\odot}$ \\
$f_{25 \mu \mathrm{m}} / f_{60 \mu \mathrm{m}}{ }^{c}$ & 0.18 \\
$M_{\mathrm{H}_{2}}{ }^{d}$ & $5.1 \times 10^{10} M_{\odot}$ \\
$L_{\mathrm{IR}} / M_{\mathrm{H}_{2}}\left(L_{\odot} / M_{\odot}\right)^{d}$ & 7.8 \\
\hline
\end{tabular}

${ }^{a}$ Coordinates refer to the northeastern nucleus of VV 114E.

${ }^{b}$ We use $H_{0}=75 \mathrm{~km} \mathrm{~s}^{-1} \mathrm{Mpc}^{-1}, q_{\mathrm{o}}=0.5$

${ }^{c}$ From Soifer et al. (1987).

${ }^{d}$ Based on Yun et al. (1994).

\section{Observations, reduction and analysis}

\subsection{The data}

VV 114 was observed with the ISOCAM camera (Cesarsky et al. 1996), a $32 \times 32$ pixel array on-board the Infrared Space Observatory (Kessler et al. 1996). The data were obtained on 6 January 1998 in low-resolution spectro-imaging mode with the Continuously Variable Filter (CVF) covering the full 5.1$16.3 \mu \mathrm{m}$ wavelength range with a spectral resolution between 30 and 40 . We used a $2.1 \mathrm{~s}$ integration per CVF frame spending a total of $2.5 \mathrm{hrs}$ on source. The pixel size was $1.5^{\prime \prime}$ creating a field of view of $48^{\prime \prime} \times 48^{\prime \prime}$, and the full-width at half-maximum $(F W H M)$ of the point spread function (PSF) varied between $4^{\prime \prime}$ and $5^{\prime \prime}$. In order to better study the low surface brightness of the MIR emission with a better signal to noise ratio than what can be achieved with the CVF, we also retrieved from the ISO archive $^{3}$ a $15 \mu \mathrm{m}$ ISOCAM image of the galaxy obtained using the wide $L W 3(12-18 \mu \mathrm{m})$ filter. This $3 \times 3$ raster map was also obtained using a pixel size of $1.5^{\prime \prime}$, a $6^{\prime \prime}$ step in both axes, and as a result it covered a $1^{\prime} \times 1^{\prime}$ field encompassing the whole optical extent of the galaxy (see Fig. 1).

The data were reduced using the CAM Interactive Analysis $\left(\mathrm{CIA}^{4}\right)$ software following the standard procedure described in detail by Starck et al. (1999). In brief we performed: 1) the subtraction of the dark current, taking into account the observing time parameters, 2) the cosmic-ray removal by applying a wavelet transform method, and 3) the correction of detector memory effects using the Fouks-Schubert's method (Coulais $\&$ Abergel 2000). The sky was subtracted using the emissionfree regions of the detector outside the galaxy. The differential pixel-to-pixel response of the array was corrected using flatfield images taken as part of the ISOCAM calibration. Finally, the effects of jitter were also corrected. These effects result from the combination of the satellite tracking motion $\left(\sim 1^{\prime \prime}\right.$ in amplitude), the continuous translation of the source on the detector as a function of the observed wavelength, which is

\footnotetext{
3 The ISO archive is available at www. iso. vilspa.esa.es/ida/

4 CIA is a joint development by the ESA astrophysics division and the ISOCAM consortium.
}

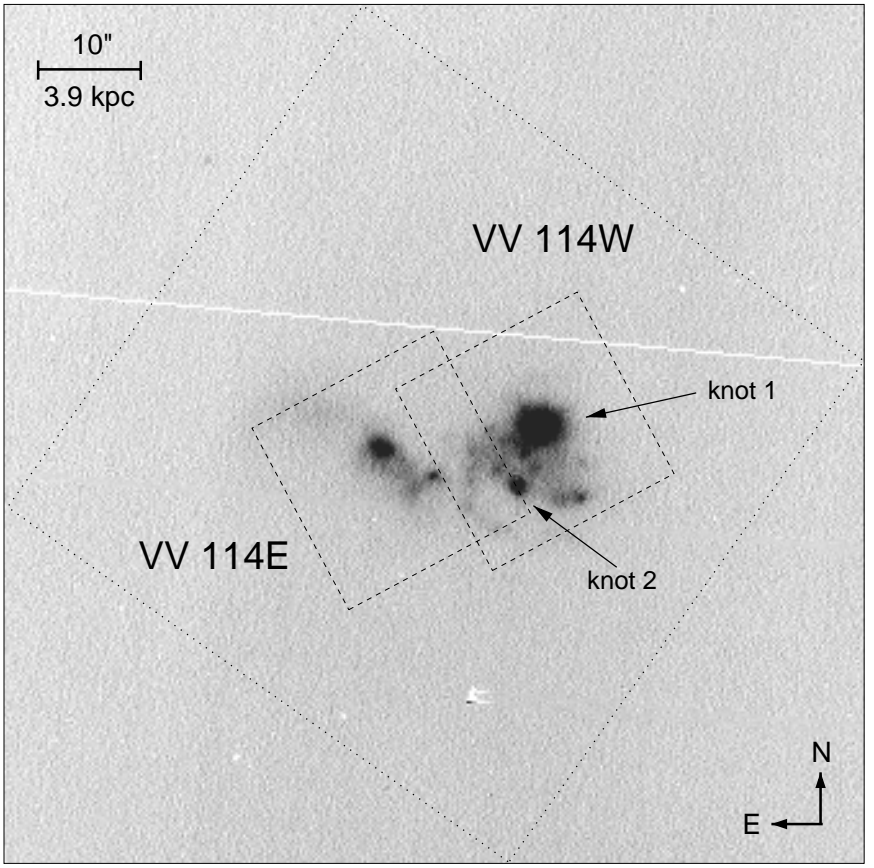

Fig. 1. An I-band image of VV 114 obtained from the CFHT archive. The two interacting galaxies, as well as the regions knot 1 and 2 of Knop et al. (1994) are marked. The field of view of the ISOCAM $15 \mu \mathrm{m} L W 3$ image is shown with the dotted square, while the two NICMOS pointings by Scoville et al. (2000) which were used to construct the mosaic presented in Fig. 2 are indicated with the dashed line. The scale and orientation of the frame are also displayed.

an intrinsic feature of the ISOCAM CVF optics, and the discontinuous shift of the target when changing from one CVF sector to another. To perform the jitter correction, all frames were resampled with a smaller pixel size and aligned with one another using the brightest peak of MIR emission observed in each frame.

\subsection{Astrometry}

Due to the absolute pointing uncertainty of the satellite, the rather small field of view of our MIR images and the lack of bright stars in the field, special care had to be taken to define the proper astrometry of our observations. For this purpose, we used the diffraction-limited images of VV 114 recently obtained by Soifer et al. (2001) with the MIRLIN mid-infrared camera (Ressler et al. 1994) on the Keck II telescope. These high resolution data indicate that 1 ) the maximum of intensity detected at $12.5 \mu \mathrm{m}$ originates from the core of VV $114 \mathrm{E}$, 2) the double-nucleus morphology of VV 114E observed at NIR and radio wavelengths is also present in the mid-infrared, and 3) both nuclei (referred as VV 114 $\mathrm{E}_{\mathrm{NE}}$ and VV 114 $\mathrm{E}_{\mathrm{SW}}$ ) have approximately the same flux at $12.5 \mu \mathrm{m}$ (see Fig. 1a of Soifer et al. 2001). Given their small angular separation of 1.6", these two sources can not be directly resolved in our data and therefore we set the peak observed in the ISOCAM images between the two (see Fig. 2b). We note that Soifer et al. (2001) reported sub-arcsec scale discrepancies regarding the spatial 
coincidence of these two nuclei as seen at NIR, MIR and radio wavelengths. These potential misalignments though are much smaller than the FWHM of the ISOCAM PSF and do not significantly affect our astrometry and analysis. We did not make any modification to the roll angle of our maps since it is unlikely to suffer from systematic errors given its observed performance over the ISO mission. In fact no uncertainties in the rotation angle were ever reported in ISOCAM deep surveys where they would have been easily observed due to the large number of detections over large spatial scales. We estimate that our final absolute astrometry is accurate to $\sim 0.5^{\prime \prime}$.

\subsection{Photometry}

The $15 \mu \mathrm{m}$ image of VV 114 (see Figs. 2a and 2b), taken with the $L W 3$ filter, shows that the MIR emission can be roughly decomposed into a bright peak at the nuclear region of VV 114E and a more extended component towards VV 114W. This diffuse emission, which was not detected in the MIRLIN images of Soifer et al. (2001), has an average surface brightness of $1.3 \mathrm{mJy} \operatorname{arcsec}^{-2}$. It is not particularly pronounced at the optical center of the western galaxy, but it presents a peak of $2.2 \mathrm{mJy} \mathrm{arcsec}^{-2}$ along the interacting region between the two galaxies (marked as knot 2 by Knop et al. 1994, see also Fig. 1). Even though the authors set a slightly higher upper limit of $1 \mathrm{mJy} \operatorname{arcsec}^{-2}$ for it, this difference can be understood as due to the higher sensitivity and larger pixel size of our ISOCAM maps.

In the analysis of our data on VV 114E, a substantial effort was devoted to determine whether the MIR ISOCAM profile can be attributed only to the double nucleus at its center, or if emission from an additional component associated with an extended circumnuclear region is also present. For this purpose, we modeled the observed profile with a combination of two point sources simulating the two nuclei of VV 114E observed by Soifer et al. (2001). As a basis for our model we used a series of theoretical PSFs calculated for the specific configuration of ISOCAM and for various offsets of the PSF maximum relative to the pixel centers on the grid of the detector (Okumura 1999). Taking into account 1) the pixel scale, 2) the orientation angle of our maps, and 3) the position angle of the two nuclei as well as their angular separation of 1.6", we thus obtained a collection of simulated profiles for each wavelength of the CVF. We then applied an iterative fitting method using a $\chi^{2}$ test to select the best simulation available. Since the relative contribution of VV $114 \mathrm{E}_{\mathrm{NE}}$ and VV $114 \mathrm{E}_{\mathrm{SW}}$ is not accurately known over the entire wavelength range of the CVF, we assumed that the two sources contribute the same level of emission throughout the 5-16 $\mu \mathrm{m}$ range, and compared the result with the extreme situation where all emission originates from VV $114 \mathrm{E}_{\mathrm{SW}}$. In both cases we found that for the best simulated profile, the fit can not account for all emission from VV 114E. The observed MIR excess is present over the whole $5-16 \mu \mathrm{m}$ range and can be attributed to the contribution from an extended component surrounding the two nuclei over an area of a few $100 \mathrm{pc}$. Before attempting to quantitatively separate this extended emission from the one originating from the two nuclei, we wanted to ensure that the former is not artificially produced by a wrong correction of the jitter effect in our data. To achieve this, we used the recorded telemetry information of the telescope, and simulated how the profile of a single PSF would widen if no jitter correction was applied. We found that the effect is noticeable only at short wavelengths, and that the error on the measured flux is negligible (less than $1 \%$ ) provided that an aperture of at least $3 \times 3$ pixel is used. We therefore chose to measure the intensity from the double nucleus by integrating the flux over a fixed aperture of $3 \times 3$ pixels $\left(4.5^{\prime \prime} \times 4.5^{\prime \prime}\right)$. Using the best simulated profiles obtained with the $\chi^{2}$ method described earlier, we found that the excess due to the extended emission around the nucleus of VV $114 \mathrm{E}$ in the aforementioned aperture is very small at long wavelengths $(\sim 10 \%$ beyond $12 \mu \mathrm{m})$, but varies between 30 and $60 \%$ at $5 \mu \mathrm{m}$ depending on the assumed relative contribution of the two nuclei. Finally, we restituted the spectral energy distribution of the double nucleus by applying an aperture correction to take account of the spatial extent of the PSF. This correction was computed assuming a $4.5^{\prime \prime} \times 4.5^{\prime \prime}$ aperture and a single point source at the center of VV 114E. Using our simulations described above, it can be shown indeed that the fraction of flux found outside of the above apertures is only weakly sensitive to the fact that VV 114E has two nuclei separated by $\sim 1.6^{\prime \prime}$.

We also compared our observed spectral energy distribution (SED) with the broad-band photometry of the central region of VV 114E as derived by Soifer et al. (2001). This was performed by integrating our spectrum over the appropriate wavelength ranges, using the profile of the MIRLIN filters. Our "MIRLIN equivalent" broad band fluxes in all five bands are in good agreement with those observed by Soifer et al. (2001) with an averaged uncertainty of $10 \%$. This consistency indicates that our assumptions and analysis resulted in a reliable MIR spectrum from the nucleus of VV 114E. Furthermore, our data indicate that VV 114E accounts for only $40 \%$ of the total MIR flux of VV 114 detected by ISOCAM. This also follows the observation by Soifer et al. (2001) that a substantial fraction of the IRAS $12 \mu$ m flux $^{5}$ is not detected in their data, and may originate from a more diffuse component, which is in fact detected by ISOCAM. Integrating over the full spatial extent of VV 114E we find that the total extended emission in VV 114E accounts for $35 \%$ of the flux beyond $12 \mu \mathrm{m}$, and reaches 50 to $70 \%$ at shorter wavelengths. To examine the spatial distribution of that diffuse component, we used the high signal to noise $15 \mu \mathrm{m}$ image and removed the contribution of the double nucleus by subtracting the best simulations selected with our $\chi^{2}$ test as mentioned above. This revealed a secondary peak of emission $3^{\prime \prime}$ west of the double nucleus of VV 114E, which is totally inconspicuous in the images dominated by the strong flux emitted by the core of the eastern galaxy. The maximum of its surface brightness is $\sim 2 \mathrm{mJy} \mathrm{arcsec}^{-2}$, and interestingly it coincides spatially with the peak of $\mathrm{CO}$ emission (Yun et al. 1994). Yet, the reality of this feature remains somewhat uncertain since it depends on the simulated profile used for the removal of the double nucleus. Moreover, it does not have any

${ }^{5}$ See Appendix A for a discussion on the IRAS measurements. 
counterpart at NIR or radio wavelengths, and may likely result from an artifact in our PSF subtraction.

Finally using our MIR spectrum, we calculated the equivalent flux densities corresponding to the typical ISOCAM broad-band filters for several components of VV 114. Taking account of the filter transmission curves, we integrated the SED from the CVF to obtain energy fluxes in $\mathrm{W} \mathrm{m}^{-2}$. The conversion to derive the flux densities in mJy was performed assuming an intrinsic spectral shape $f_{v}=$ cte. The total intensity for the two galaxies was obtained by integrating the MIR emission over the full spatial extent of the galactic disks as seen in our data. We note that within $1 \sigma$ of our photometric uncertainty, the flux measured by the $L W 10$ filter, an equivalent to the $12 \mu$ m IRAS filter, accounts for nearly $100 \%$ of the IRAS flux, the exact value of which we discussed in Appendix A. The flux from the central region of VV 114E was derived as explained earlier, and the one from the peak of the extended emission located southeast of VV $114 \mathrm{~W}$ was measured over a region of $5^{\prime \prime}$ in diameter centered on knot $2\left(\alpha=01^{\mathrm{h}} 07^{\mathrm{m}} 46.7^{\mathrm{s}}, \delta=-17^{\circ} 30^{\prime} 27^{\prime \prime}\right.$, J2000). Our results are presented in Table 2 . The $1 \sigma-$ rms noise in our images created from the CVF by integrating over the $5-8.5 \mu \mathrm{m}$ (equivalent $L W 2$ filter) and $12-16 \mu \mathrm{m}$ wavelength range are 150 and $230 \mu \mathrm{Jy} \operatorname{arcsec}^{-2}$ respectively. Clearly, these images are not as deep as the one obtained directly with the broad-band $L W 3$ filter in which we measured a noise level of $70 \mu \mathrm{Jy} \operatorname{arcsec}^{-2}$. This was to be expected since the bandpass of the CVF is much narrower than the width of the broadband filters. The relative photometric uncertainty on the MIR SED varies from $10 \%$ at $5 \mu \mathrm{m}$ up to $15 \%$ at $16 \mu \mathrm{m}$. The major sources of errors are due to the non-perfect correction of the detector memory effect. Based on our experience with ISOCAM data, we estimate that an uncertainty of $25 \%$ for the absolute photometry constitutes a conservative upper limit, which is typical for well detected extended sources.

\subsection{Mapping of the UIB emission}

As we will discuss in more detail in Sect. 3.2, it is widely accepted that the various Unidentified Infrared Bands (UIBs) detected in MIR spectra of galaxies are produced in photodissociation regions (PDRs) and consequently are directly associated with the regions of star formation in galaxies (see Rigopoulou et al. 1999; Laurent et al. 2000; Helou et al. 2000). Their spatial distribution could then be used to depict more accurately the regions undergoing star forming activity even in areas where extinction from dust is high. We thus extracted the UIB emission from our CVF spectral cube and produced images of their spatial distribution across VV 114, following the same method as in Le Floc'h et al. (2001). We identified the 6.2, 7.7 and $11.3 \mu \mathrm{m}$ bands in all observed positions (pixels) on the galaxy and subtracted the underlying continuum emission (see Fig. 3 for an illustration of the method). Since on galactic scales the relative strength of the different UIBs does not vary substantially, we co-added the corresponding images to produce a single higher signal to noise map of the integrated UIB emission. We decided not to include the $8.6 \mu \mathrm{m}$ feature which is highly sensitive to the strength of the $9.7 \mu \mathrm{m}$ silicate absorption band. The $12.7 \mu \mathrm{m}$ feature was also excluded since it is contaminated by the [NeII] line at $12.8 \mu \mathrm{m}$ (see Sect. 3.2.1).

\section{Results}

\subsection{Spatial distribution of the MIR emission}

In Fig. 2a, we overlay in contours this UIB map on a $15 \mu \mathrm{m}$ image of VV 114 obtained with the $L W 3$ filter while in Figs. $2 \mathrm{~b}$ and c, we present a mosaic of the two $1.1 \mu \mathrm{m} \mathrm{J}$-band images of VV 114 taken with HST/NICMOS ${ }^{6}$ superimposed with the contours of the $15 \mu \mathrm{m}$ and UIB emission respectively. One can see that the UIB emission seems to be more closely restricted to the active regions of the galaxy than the widespread distribution of hot dust seen in the $15 \mu \mathrm{m}$ image. This apparent lack of UIB features in the outskirts of VV 114 is most probably due to a lower sensitivity of our UIB maps.

The power of infrared to probe deeper into the embedded regions of galaxies is particularly enlightening in the case of VV 114E, the activity of which becomes apparent in the thermal MIR even though it is rather inconspicuous in the optical. We clearly see that this source dominates the MIR emission of VV 114 (see Fig. 2a), and roughly accounts for $40 \%$ of the total flux observed. The fact that VV $114 \mathrm{E}$ has an $L W 3$ to $K$-band ratio of $\sim 16$ which is larger than the 1-10 range of values found in late type galaxies (Boselli et al. 1997), suggests a higher than average hot dust content compared to its stellar mass. Strong UIB features are also seen in its spectrum (see Fig. 2c) further confirming that an important fraction of the flux coming from the central regions of the eastern galaxy originates from a powerful circumnuclear starburst (see also Sect. 3.2.2). Our findings support similar conclusions derived by Doyon et al. (1995) based on NIR spectroscopic observations. Note that the intense star forming activity in the inner core of VV 114E had also been inferred from the strong $8.4 \mathrm{GHz}$ radio emission (Condon et al. 1991), which coincides with the bright NIR peaks (Knop et al. 1994), as well as the large quantity of molecular gas in the vicinity of VV 114E (Yun et al. 1994).

Contrary to VV 114E, the MIR emission from VV 114W is weaker and more diffuse. Surprisingly, there is no enhancement of the MIR flux near its nucleus. Instead, the dust emission seen with ISOCAM peaks $5^{\prime \prime}$ to the southeast, close to the bright $\mathrm{H}_{\text {II }}$ region denoted as knot 2 by Knop et al. (1994). This secondary peak at knot 2 is even more pronounced in our UIB map and is also visible in the $3.2 \mu \mathrm{m}$ image of Soifer et al. (2001) which sampled emission from the weaker $3.3 \mu \mathrm{m}$ UIB feature. Knot 2 also coincides with a local maximum of the radio emission at $1.49 \mathrm{GHz}$ (Condon et al. 1990), and has an $\mathrm{H} \alpha$ equivalent width of $430 \AA$, six times wider than what is observed in the nucleus of VV $114 \mathrm{~W}$, a clear evidence of massive extranuclear star formation activity (Knop et al. 1994). A large number of young star clusters and compact $\mathrm{H}_{\text {II }}$ regions were also detected in the NIR throughout the same

\footnotetext{
6 Individual frames are available at the NASA Extragalactic Database hosted in the web server of IPAC, see also Scoville et al. (2000).
} 
Table 2. ISOCAM broad-band photometry of VV 114.

\begin{tabular}{lccccccc}
\hline \hline & \multicolumn{3}{c}{ ISOCAM flux in broad-band filters (mJy) } \\
& $L W 2$ & $L W 3$ & $L W 4$ & $L W 6$ & $L W 7$ & $L W 9$ & $L W 10^{a}$ \\
Source & $(5-8.5 \mu \mathrm{m})$ & $(12-18 \mu \mathrm{m})$ & $(5.5-6.5 \mu \mathrm{m})$ & $(7-8.5 \mu \mathrm{m})$ & $(8.5-10.7 \mu \mathrm{m})$ & $(14-16 \mu \mathrm{m})$ & $(8-15 \mu \mathrm{m})$ \\
\hline VV 114E (nucleus) & 136 & 351 & 96 & 242 & 105 & 379 & 198 \\
VV 114W (knot 2) & 28 & 88 & 13 & 62 & 35 & 85 & 58 \\
VV 114 (diffuse) & 256 & 241 & 111 & 296 & 180 & 186 & 264 \\
\hline VV 114 (total) & 420 & 680 & 220 & 600 & 320 & 650 & 520 \\
\hline
\end{tabular}

${ }^{a}$ equivalent to IRAS $12 \mu \mathrm{m}$ filter.

Note: Fluxes in the $L W 3$ filter were measured directly in the $L W 3$ broad-band image retrieved from the ISO archive. Equivalent fluxes in the other filters were obtained from the CVF data, those of VV 114E (nucleus) and VV 114W (knot 2) being derived from their MIR SED respectively presented in Figs. 3 and 4. The diffuse emission was deduced after subtracting the contributions of VV 114E (nucleus) and knot 2 to the total fluxes measured over the full spatial extent of the merger.
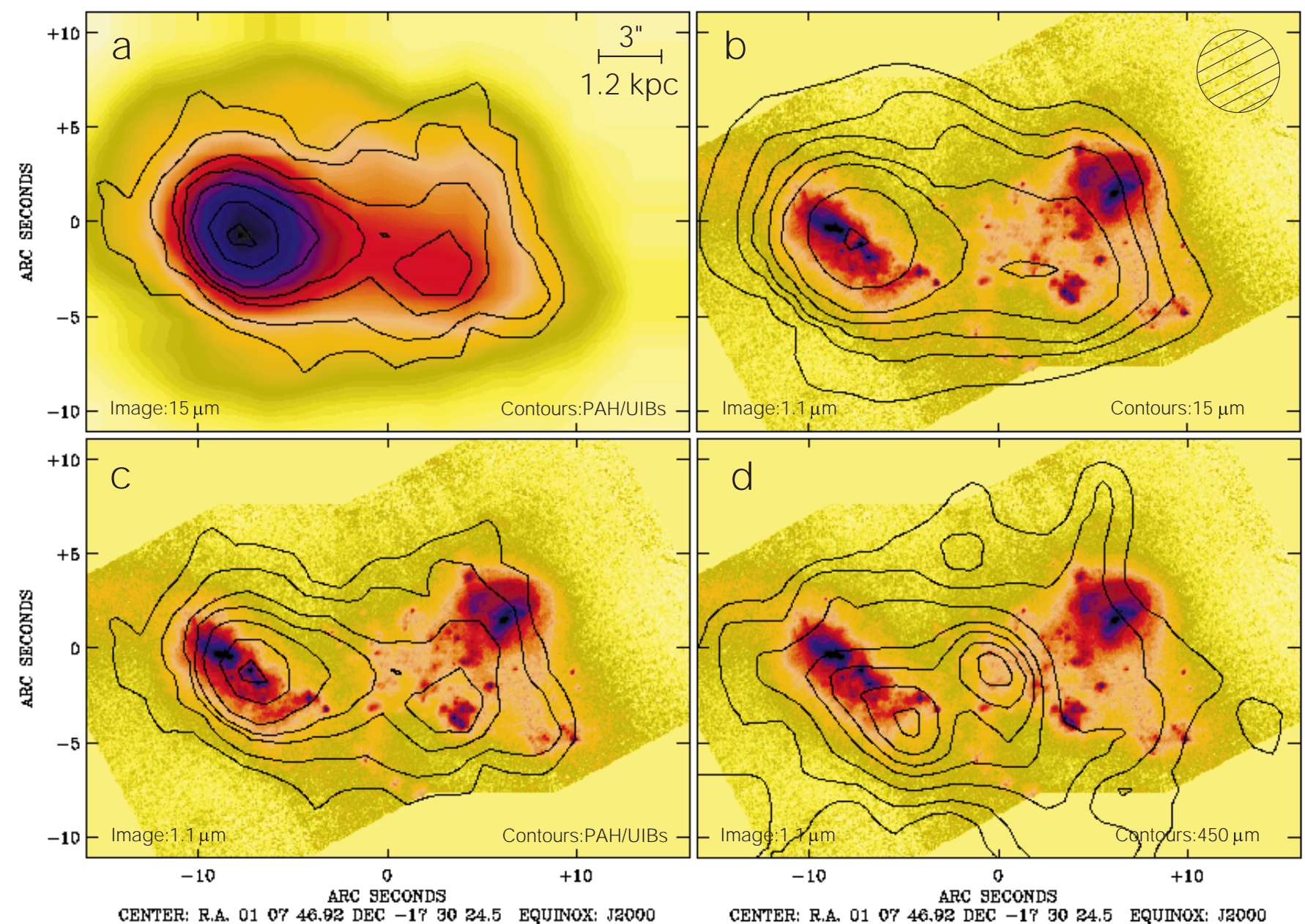

Fig. 2. a) The ISOCAM $15 \mu \mathrm{m}$ image of VV 114 taken with $L W 3(12-18 \mu \mathrm{m})$ filter with an overlay of the integrated UIB emission at $6.2,7.7$ and $11.3 \mu \mathrm{m}$ (see Sect. 2.4). The image scale is displayed with a bar of $1.2 \mathrm{kpc}$ ( $3^{\prime \prime}$ ) at the top-right corner. b) The HST/NICMOS $J$-band image of VV 114 from Scoville et al. (2000) with the overlay of the $L W 315 \mu \mathrm{m}$ image presented in a). The contour levels are $0.45,0.65,0.90,1.35$, $2.10,4.45$ and $8.45 \mathrm{mJy} \mathrm{arcsec}^{-2}$. We have also indicated the $F W H M$ of the ISOCAM PSF at $12 \mu \mathrm{m}$. Note that the warm dust emission is much more extended than the nuclei of the galaxies. c) The same $J$-band image presented in b) but overlayed with the UIB contours of a). Note how the secondary UIB peak is not found in VV 114W but instead it coincides with knot 2 of Knop et al. (1994). d) An overlay of the SCUBA $450 \mu \mathrm{m}$ emission detected by Frayer et al. (1999) onto the NICMOS image presented in b) and c). The contours are 150, 225, 338, 450, 525, 600 and $675 \mathrm{mJy}$ beam $^{-1}$ while the beam size is $\sim 6^{\prime \prime}$. We observe that, similar to the CO emission, most of the cold dust is found in the area between the galaxies suggesting that there is a considerable reservoir of gas to fuel the star formation activity in the system. 
area (Scoville et al. 2000). As a whole though, VV 114W has an $L W 3 / \mathrm{K}$ ratio $\sim 11$ slightly higher than that of normal galaxies, and accounts for $\sim 35 \%$ of our observed total MIR flux.

Another striking feature in our maps is the spatial extent of the diffuse MIR emission. This is clearly noticeable in the $15 \mu \mathrm{m}$ image of VV 114 (see Fig. 2b). Its distribution is rather smooth with an elliptical shape surrounding the central regions of the two merging galaxies over more than $10 \mathrm{kpc}$. This is in stark contrast to what is observed in most LIRGs and ULIRGs which typically harbor very compact circumnuclear starbursts responsible for nearly $80-100 \%$ of the energy emitted in the mid-infrared (i.e. Soifer et al. 2000, 2001; Charmandaris et al. 2002b). As was already noted by Soifer et al. (2001) though, VV 114 is an exception to this trend since its $12 \mu \mathrm{m}$ and NIR curves-of-growth are quite similar. We explore the implications of this finding in Sect. 4.

At kpc scales, the diffuse and extended emission from hot dust particles as seen in our data closely follows the distribution of the molecular gas detected in the $\mathrm{CO}$ interferometric map of Yun et al. (1994). The CO emission reveals a fairly extended and massive molecular gas complex at the center of the merger, distributed along a bar joining the two galaxies. Two tail-like features extend from the extremities of this bar, one to the North of VV 114W, and the other to the South of VV 114E. This morphological correspondence between CO and MIR emission in tails or spiral arms has already been observed both in normal and in interacting galaxies and AGN (Mirabel et al. 1999; Wilson et al. 2000; Le Floc'h et al. 2001) and is to be expected as the molecular gas is the fuel of star formation which in turn heats the dust to emit in the MIR.

Recently, submillimeter observations of VV 114 by Frayer et al. (1999) at $450 \mu \mathrm{m}$ and $850 \mu \mathrm{m}$ with SCUBA have also revealed a cold dust emission centered in the overlap regions between the two interacting components and extended over $12 \mathrm{kpc}$. As we can see from Fig. 2d, despite the large SCUBA beam (6" at $450 \mu \mathrm{m})$ the cold dust follows the same overall distribution as our ISOCAM maps, but the two peaks of the $450 \mu \mathrm{m}$ emission are slightly displaced relative to the MIR. This can be interpreted by temperature gradients within the dust reservoir which is heated by both the nucleus of VV 114E and the star-forming source at knot 2 . The distribution of the cold dust seen at $850 \mu \mathrm{m}$ also correlates well with the CO emission given the uncertainty $\left( \pm 2^{\prime \prime}\right)$ of the SCUBA map, and seems to peak in the overlap region of the merging galaxies just to the southwest of VV 114E. However, determining the location where the bolometric luminosity of VV 114 mainly originates is not straight forward. According to the models of the FIR emission presented in Fig. 2 of Frayer et al. (1999), most of the energy output in this merger is radiated between 60 and $200 \mu \mathrm{m}$. We note that the emission probed by SCUBA only accounts for less than one tenth of the total luminosity and is similar to the contribution of the MIR emission traced by ISO at $15 \mu \mathrm{m}$. This suggests that the $60-200 \mu \mathrm{m}$ emission is probably concentrated between the peaks of the MIR and submillimeter emission, and thus the bolometric luminosity of the system should be more closely associated with the eastern component of VV 114.

\subsection{Spectral properties}

\subsubsection{The Mid-infrared SED of VV 114W}

The spectral shape of the MIR emission throughout the extent of VV $114 \mathrm{~W}$ was found rather constant across the entire disk as well as on the overlap region towards the eastern component, varying only in intensity. In Fig. 3 we present the spectrum of a $5^{\prime \prime}$-diameter aperture centered on the brightest peak of emission in VV 114W. As mentioned earlier, this is the location of the so-called knot 2, which has the widest $\mathrm{H} \alpha$ equivalent width of the galaxy. The MIR spectrum is typical of what is observed in other galaxies which are actively forming stars (i.e. Laurent et al. 2000 and references therein). As it is known, the spectrum can be decomposed mainly into the contribution of the Unidentified Infrared Bands (UIBs) clearly detected at 6.2, $7.7,8.6,11.3$ and $12.7 \mu \mathrm{m}$, and that of a steeply rising continuum longward of $10 \mu \mathrm{m}$. The UIBs are generally attributed to $\mathrm{C}=\mathrm{C}$ and $\mathrm{C}-\mathrm{H}$ vibrations in Polycyclic Aromatic Hydrocarbon molecules (PAHs, see Léger \& Puget 1984; Li \& Draine 2001) which are stochastically heated by UV and optical radiation, and since they are typically found in PDRs they are often used to trace quiescent or more active star formation (Helou et al. 2000). The rising continuum on the other hand, is the most prominent feature detected in MIR spectra of galactic $\mathrm{H}_{\text {II }}$ regions and galaxies harboring intense star forming activity. It is thought to be produced by small dust particles with radius less than $10 \mathrm{~nm}$ (Very Small Grains - VSGs - see Désert et al. 1990), which are heated up to temperatures $\sim 200-1000 \mathrm{~K}$ by the strong radiation field from hot young stars, and therefore it is considered as a good tracer of massive star formation activity. The presence of this continuum in our spectrum - though as we will see rather weak compared to VV 114E - confirms that vigorous star formation is underway in the overlap region of the merger, where the streaming motions and shocking of gas clouds due to the interaction are probably the strongest. This is in contrast to what one observed in the MIR spectra of quiescent spiral galaxies where the contribution from the VSGs is generally much lower (Roussel et al. 2001). The overall extinction in the area, as measured by the relative strength of the 6.2 and $11.3 \mu \mathrm{m}$ UIB features (see Sect. 3.2.2 for a description of the method used), is small and the ISOCAM star formation activity indicator as traced by the $L W 3 / L W 2$ color ratio is just above $\sim 1.4$ (see Laurent et al. 2000).

Several ionic emission lines are also visible in the spectrum. At $7 \mu \mathrm{m}$, we detect [ArII], which has a rather low ionization potential $\left(E_{\mathrm{p}}=16 \mathrm{eV}\right)$ and is typically found in starburst environments (Sturm et al. 2000). Given our low spectral resolution, the $12.8 \mu \mathrm{m}$ [NeII] line $\left(E_{\mathrm{p}}=22 \mathrm{eV}\right)$ is blended with the $12.7 \mu \mathrm{m}$ UIB feature and its strength can not be accurately determined. However, ISOCAM CVF observations of many regions with moderate star forming activity and low extinction (i.e. Boulanger et al. 1996) indicate that the 12.7 and $11.3 \mu \mathrm{m}$ UIB features scale with one another. The fact that the $12.7 \mu \mathrm{m}$ emission in VV $114 \mathrm{~W}$ is fairly strong suggests that a considerable fraction of the feature flux is due to the [NeII] line. This would be expected given the high $\mathrm{H} \alpha$ flux of knot 2, a clear evidence of massive extranuclear star formation activity. 


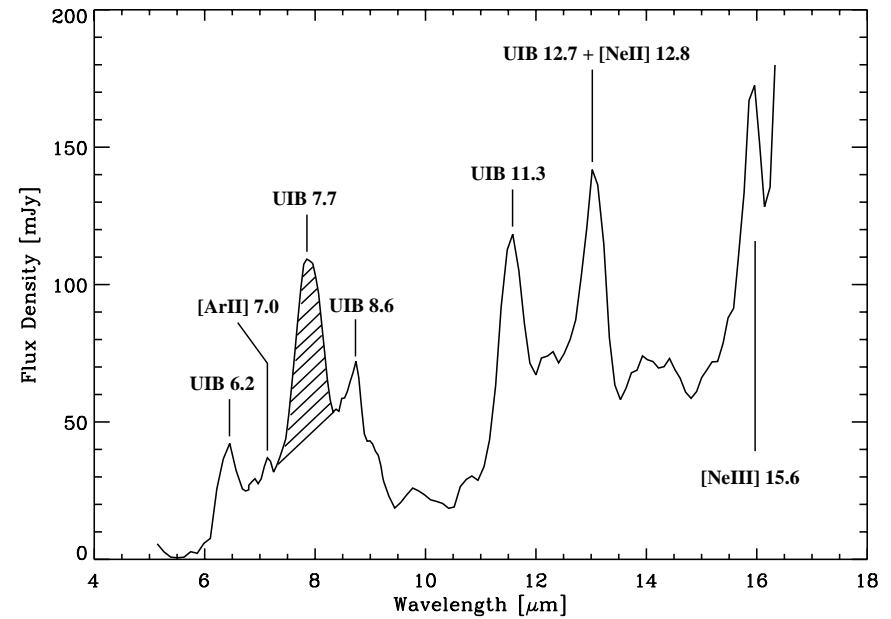

Fig. 3. Mid-infrared spectrum of knot 2 in VV 114W obtained with an aperture of $5^{\prime \prime}$ in diameter, centered on the peak of emission located near the bright $\mathrm{H}$ II region southeast of the nucleus of VV 114W $\left(\alpha=01^{\mathrm{h}} 07^{\mathrm{m}} 46.7^{\mathrm{s}}, \delta=-17^{\circ} 30^{\prime} 27^{\prime \prime}, \mathrm{J} 2000\right)$. The flux of the UIB feature at $7.7 \mu \mathrm{m}$ used to create the UIB map presented in Fig. $2 \mathrm{~b}$ was measured integrating the hatched area as illustrated in the figure. A similar technique was applied to the other features at 6.2 and $11.3 \mu \mathrm{m}$.

The [NeII] line has also been clearly observed with the larger aperture of ISO-SWS (Thornley et al. 2000), which provides further confidence on the reliability of our detection. We finally observe an apparent bright feature at $15.6 \mu \mathrm{m}$, which we attribute to [NeIII]. The profile of that feature as well as the fact that this line was also observed with ISO-SWS, lead us to believe that our detection is reliable, even though the obvious memory effects of the detector make a determination of its flux problematic.

\subsubsection{The MIR spectrum of the nuclear region in VV 114E}

As discussed in Sect. 2.3, the MIR emission from the central region of the eastern galaxy is dominated by the contribution of its nucleus over the full wavelength range of the CVF. In Fig. 4 we present the spectrum of the central $4.5^{\prime \prime} \times 4.5^{\prime \prime}$ $(1.7 k p c \times 1.7 k p c)$ region of VV $114 \mathrm{E}$, after correcting it via a scaling factor that takes into account the extended flux of the PSF lying outside the aperture. As in VV $114 \mathrm{~W}$, we clearly detect the presence of the UIB features as well as the [NeII] and [NeIII] lines. However, comparing the spectra of the two galaxies, we note that in VV 114E the relative strength of the 8.6 and $11.3 \mu \mathrm{m}$ features compared to the UIBs at 6.2, 7.7 and $12.7 \mu \mathrm{m}$ is much lower, which implies a higher silicate absorption at $9.7 \mu \mathrm{m}$ in the eastern galaxy. The latter is also suggested by the $15 \mu \mathrm{m}$ to $9.7 \mu \mathrm{m}$ flux ratio, which for VV $114 \mathrm{E}$ is $\sim 8$, nearly 4 times that of normal galaxies (see $\mathrm{Xu}$ et al. 2000). The increased extinction towards VV 114E is also apparent as the fraction of the total flux originating from VV 114E reaches $65 \%$ of the total shortward of $9 \mu \mathrm{m}$ and longward of $12 \mu \mathrm{m}$, but falls to only $50 \%$ near the silicate band (see Table 2 and Fig. 5). To estimate the extinction in the central region of VV 114E, we reproduced the relative strength of the UIB
Table 3. Extinction in the central region of VV 114E.

\begin{tabular}{lcc}
\hline \hline Extinction law & $A_{v}$ & $\tau$ at $10 \mu \mathrm{m}$ \\
\hline Draine (1989) & $4 \mathrm{mag}$ & 0.33 \\
Mathis (1990) & $11 \mathrm{mag}$ & 0.60 \\
Dudley \& Wynn-Williams (1997) & $5 \mathrm{mag}$ & 0.28 \\
Lutz (1999) & $6 \mathrm{mag}$ & 0.83 \\
Li \& Draine (2001) & $5 \mathrm{mag}$ & 0.41 \\
\hline
\end{tabular}

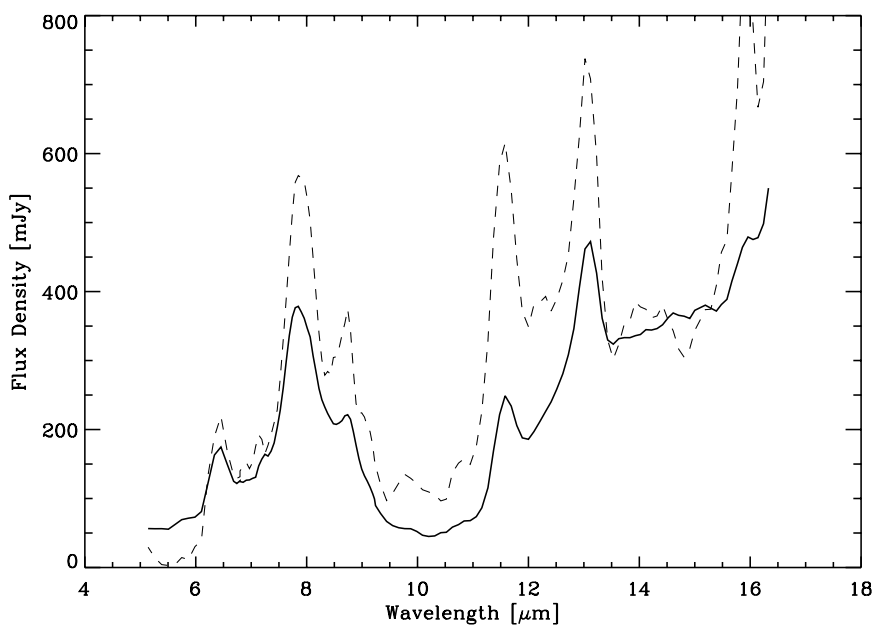

Fig. 4. The mid-infrared spectrum of the central region of VV 114E using an aperture of $4.5^{\prime \prime} \times 4.5^{\prime \prime}$, taking into account the variable size of the PSF. For comparison, we have reproduced the spectrum of VV 114W (dashed-line) normalized to the rest frame 14-15 $\mu \mathrm{m}$ continuum. Note the difference in the slope of spectra as well as the higher extinction in VV 114E.

features at 6.2 and $11.3 \mu \mathrm{m}$ using the observed spectrum of the M 51 disk extinguished by a screen model with different extinction laws. This approach is justified since in unobscured star forming regions, UIB features in the $5-13 \mu \mathrm{m}$ range are known to present canonical spectral properties between one another (Dale et al. 2001; Roussel et al. 2001). The various extinction laws were chosen to explore the effects of a variable strength in the $9.7 \mu \mathrm{m}$ silicate band, and they also differ in their level of extinction between 4 and $8 \mu \mathrm{m}$. The results obtained are summarized in Table 3. In spite of the apparent variations between the different measurements, all values of $\tau$ are lower than 1 , which is consistent with an optically thin medium and making the screen model a raisonable assumption.

We note that we measure a lower extinction than Soifer et al. (2001) who derived $\tau \sim 0.7$ for the southwestern nucleus of VV 114E and $\tau \sim 2-3$ for the northeastern nucleus using the extinction law of Li \& Draine (2001) at $10 \mu \mathrm{m}$. Our lower measurements are expected since our larger ISOCAM beam samples a more diffuse optically thin dust component surrounding the two nuclei of VV 114E.

More intriguing is that VV 114E displays a rather significant continuum at short MIR wavelengths $(5-6 \mu \mathrm{m})$. This "bump" is absent in pure starburst spectra, but has already been observed between 3-6 $\mu \mathrm{m}$ in the SEDs of active galactic nuclei (Lutz et al. 1998; Mirabel et al. 1999; Laurent et al. 2000; Le Floc'h et al. 2001) and it has been proposed as a MIR diagnostic for an AGN (Laurent et al. 2000). According to the 


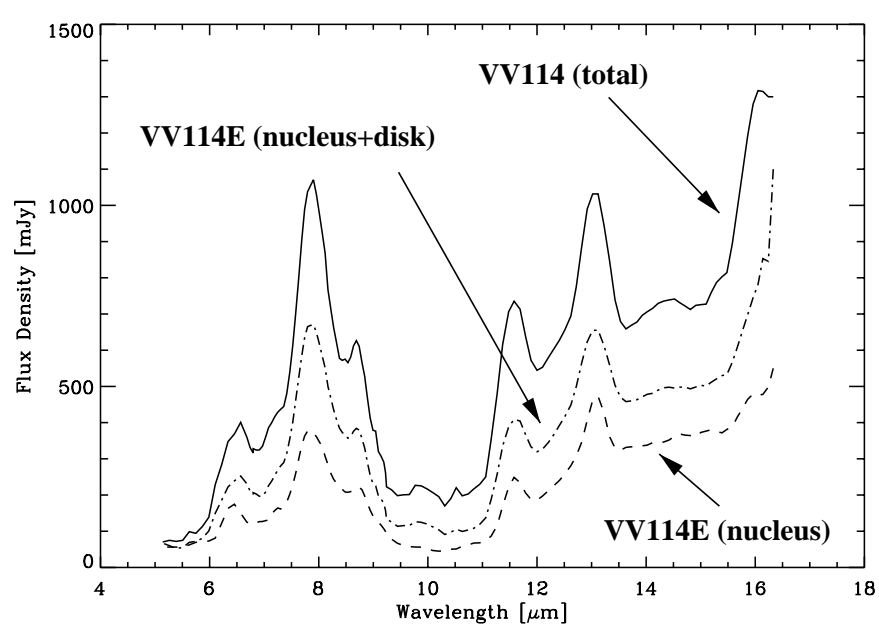

Fig. 5. The total MIR spectrum of VV 114 (solid line) integrated over the full spatial extent of the merger. The $5-16 \mu \mathrm{m}$ SED of the nucleus of VV 114E (dashed-line) and the spectrum of the whole eastern galaxy (disk+nucleus, dashed-dotted line) are also reproduced to show their relative contribution to the total emission.

unified scheme of AGNs, such a continuum is thought to be produced by very hot dust particles heated to nearly their sublimation temperature $(T \sim 1000 \mathrm{~K}$ for silicate and $T \sim 1500 \mathrm{~K}$ for graphite) within the torus of molecular gas surrounding the nucleus (Krolik 1999). The elevated continuum at $5 \mu \mathrm{m}$ in our spectrum would be consistent with emission from an enshrouded AGN. One should note however, that no other telltale signs of an active nucleus such as the $[\mathrm{NeVI}]$ and $[\mathrm{NeV}]$ lines have been detected on that galaxy, despite the rather modest nuclear extinction of the nucleus. Could it be that this "bump" is due to an ultracompact starburst instead? We discuss this possibility in the following section.

\section{Discussion}

\subsection{VV 114: The early stage of a future ULIRG}

As we mentioned earlier, ULIRGs are formed when gravitational instabilities occuring during galactic collisions, force large concentrations of gas to the central regions of the merging disks, fueling circumnuclear starbursts or enshrouded active nuclei (see Mihos \& Hernquist 1996). Observations of ULIRGs in the near and mid-infrared have revealed that a large fraction (30-100\%) of the infrared energy emitted by these objects is often contained in very compact nuclear regions with linear scales of a few 100 pc (Scoville et al. 2000; Soifer et al. 2000, 2001). Moreover, Gao \& Solomon (1999) have shown a strong correlation between the CO luminosity and the nuclear separation of merging galaxies, as well as an anti-correlation between that distance of the nuclei and the $L_{\mathrm{IR}} / M_{\mathrm{H}_{2}}$ ratio. In this context, one would expect that the ultraluminous phase would appear as the final result of the merging, once the two progenitors have nearly formed a single object. However, it has been recently reported that a population of ULIRGs may consist of widely separated $(>10 \mathrm{kpc})$ pairs of galaxies in a rather early phase of interaction (Murphy et al. 2001; Dinh et al. 2001; Meusinger et al. 2001). This finding implies that our interpretation on the mechanisms stimulating star formation during interactions needs to be re-examined. More specifically, Murphy et al. (2001) have suggested that galaxies which have sufficiently large reservoirs of molecular gas and experience more than one close encounter before their final merger, may undergo multiple stages of ultraluminous activity $\left(L_{\mathrm{IR}} \gtrsim\right.$ $\left.10^{12} L_{\odot}\right)$ triggered at each encounter of their disks while remaining in the LIRG stage during the in-between periods.

Several observational evidence lead us to believe that VV 114 is in fact in such a "quiescent LIRG" phase. The first one is the moderate separation ( $\sim 6 \mathrm{kpc}$ in projection) between the two interacting galaxies, and the very extended molecular gas distribution as revealed by the $\mathrm{CO}$ observations. The gas kinematics are also characterized by large velocity gradients and non-rotational signatures dominated by inflow/outflow radial motions, and even the distribution of the stellar light in the central regions has not yet settled into an $r^{1 / 4}$ profile (Yun et al. 1994; Scoville et al. 2000). Yet, despite the early stage of its evolution, VV 114 is already one of the brightest infrared objects in the local Universe (Soifer et al. 1987), and harbors compact nuclear and extranuclear starbursts with strong MIR emission. We note that such spots of star formation in the overlap region of interacting galaxies are not uncommon when the progenitors are late type galaxies (Mirabel et al. 1998; Gallais et al. 1999; Xu et al. 2000). Another characteristic of VV 114, yet unusual in LIRGs, is the similar curves-of-growth at 2.2 and $12.5 \mu \mathrm{m}$ in the central $1 \mathrm{kpc}$ region of VV 114E (Soifer et al. 2001). What could be the cause for this behavior? A possible explanation might be related to the fact that even though the system is highly IR luminous, it is dynamically young with its two gas rich components still violently interacting. This would imply that not only the old stellar population but large quantities of dust grains coupled to the gas originally associated with the disks of VV 114E and VV 114W before their interaction, have not yet relaxed and they are distributed around the galaxies following loops and tails in the gaseous component. The strong ionization field resulting mainly from the massive starbursts in VV 114E as well as in the overlap region, would then permeate this extended volume of gas and dust, and could cause the dust grains of smaller size to be heated sufficiently in order to emit in the thermal MIR.

The large-scale spatial coincidence between the $\mathrm{CO}$ and MIR emission shows that the conversion of molecular gas into stars is still ongoing, not only in compact regions where the gravitation potential is responsible for fueling the starburst with gas, but also in more extended areas where shocks and density enhancements induced by the dynamical perturbations of the collision may also trigger a star forming activity. Taking into account the estimated age of the collision $\left(\sim 3-4 \times 10^{8} \mathrm{yrs}\right.$, Yun et al. 1994) and the huge reservoir of gas still available $\left(5.1 \times 10^{10} M_{\odot}\right)$, it is very likely that the star formation process will continue for a least a few $10^{8}$ yrs before the two galaxies finally coalesce. As suggested by Yun \& Scoville (1995) to illustrate how the gas density and IR luminosity efficiency anticorrelate with nuclear separation in merging systems, VV 114 could evolve into a more advanced merger like Mrk 273, to finally terminate in a ULIRG similar to Arp 220. 


\subsection{Is there a buried AGN in VV114E?}

The question of whether AGN or compact starbursts are responsible for the production of the bulk of the luminosity of LIRGs/ULIRGs is a pertinent one. The fact that both phenomena usually co-exist makes quantifying their contribution rather problematic (see Laurent et al. 2000 for a discussion). Recently, large scale surveys conducted at MIR wavelengths with ISO (Lutz et al. 1998) have provided evidence that roughly $70 \%$ of the energy budget in the Universe would originate from star forming activity, though it has been suggested from submillimeter surveys that the contribution from AGNs could be actually more important (McMahon et al. 1999).

The colors of VV 114E are very red indicating a significant absorption of UV and optical light by large concentrations of dust and thus one may wonder whether or not an active nucleus, hidden in the visible, could be lurking in it. Apriori though, several observational evidence argue against it. Not only the cold IRAS color of VV $114\left(f_{25 \mu \mathrm{m}} / f_{60 \mu \mathrm{m}}=0.18\right)$ indicates a predominant star formation activity, but also the two nuclei of VV 114E show extended emission at both NIR (Knop et al. 1994; Doyon et al. 1995) and radio (Condon et al. 1991) wavelengths, which suggests that the observed continuum has a stellar origin. Moreover, the NIR spectroscopy of VV 114E indicates that the gas is photo-ionized by young OB stars rather than an AGN-like engine (Doyon et al. 1995), which reinforces the idea that the bulk of the infrared emission from the galaxy may be attributed to an episode a vigorous star formation.

However, using a $J-H$ vs. $H-K$ color diagram, it can be shown that the very central region of VV 114E exhibits near-infrared colors similar to that observed in warm LIRGs (Scoville et al. 2000). Furthermore, models fitting the NIR SED of VV 114E indicate that $50 \%$ of the $K$-band continuum observed in the two nuclei originates from hot dust emission heated to temperature $\sim 800 \mathrm{~K}$ (Doyon et al. 1995). Unfortunately, even the radio data of Condon et al. (1991) are not conclusive for the two nuclei of VV $114 \mathrm{E}$ as no high resolution radio spectral indexes which could be used to identify a synchrotron or a flat thermal origin of their emission are available. Finally, as it was shown by Soifer et al. (2001) as well as from ISOCAM data, only a fraction of the MIR emission seen by IRAS is associated with the nuclear region of VV 114. Consequently the IRAS colors do not characterize the nuclear activity in this galaxy and can not be used to exclude a dustenshrouded active nucleus in VV 114E.

In fact, our MIR spectra of VV 114E do reveal a strong emission at $5 \mu \mathrm{m}$, and a rather flat $5-16 \mu \mathrm{m}$ continuum compared to typical template ISOCAM spectra of $\mathrm{H}_{\text {II }}$ regions (Laurent et al. 2000). The specific signatures of PDRs, HiI regions and AGNs in the mid-infrared have been thoroughly studied by Genzel et al. (1998) and Laurent et al. (2000) who, using the UIB to continuum flux ratio of MIR templates to characterize each of the different types of activity, have both developed diagnostics to distinguish between starbursts and active nuclei in galaxies. Up to now, the type of MIR spectrum seen from VV 114E has only been found in AGNs and it is thus considered as a solid indicator for the presence of an active nucleus which is dominant in the MIR. Using the classification scheme of Laurent et al. (2000) it appears that an AGN could be contributing up to $40 \%$ of the MIR flux in the eastern galaxy of VV 114. Such a result supports the strength of MIR observations to unveil dusty and hidden AGNs which can not be detected at other wavelengths.

Recently, it has been proposed to combine the $3.3 \mu \mathrm{m}$ PAH emission with the $3.4 \mu \mathrm{m}$ carbonaceous dust absorption to distinguish between AGN and starburst activity in luminous galaxies (Imanishi \& Dudley 2000; Imanishi 2002). This technique could be particularly powerful in detecting buried active nuclei in LINER- or starburst-classified LIRGs/ULIRGs. It would be of great benefit if this new diagnostic could be applied to VV $114 \mathrm{E}$ so that we obtain a better view on the $2-5 \mu \mathrm{m}$ window properties of that merger. Establishing the link between what is observed in the $K$ and $L$ band and the short $(4-6 \mu \mathrm{m})$ MIR wavelengths, which were observed by ISO and will also be covered by SIRTF in the near future, is clearly essential in order to better constrain the origin of the different contributions and processes at work in this system.

\subsection{Implications for high redshift surveys}

Characterizing the physical properties of sources in the distant Universe is one of the key issues to discriminate between various models of galaxy formation. It has become increasingly accepted that understanding the properties of galaxies such as VV 114 may provide a valuable insight for this goal. As discussed earlier, this system consists indeed of two galaxies for which the presence of dust is the cause of their dramatically different SEDs. Most of the bolometric luminosity originates from VV 114E which also dominates the FIR emission from the system but is completely obscured by dust in the far- and near-UV (Goldader et al. 2002). On the other hand, the western galaxy, VV $114 \mathrm{~W}$, is bright in the UV and visible and contributes only modestly to the global infrared emission. These "antagonistic" features have important consequences for the detection as well as the apparent morphology of high redshift galaxies. If we were to place VV 114 at $z \geq 1$, the spatial resolution of the deep surveys at optical wavelengths (e.g. HDF, Williams et al. 1996) would not allow us to disentangle the two components involved. One therefore would have to rely only on its integrated properties. Goldader et al. (2002) have shown that VV 114 could be easily observed at $z \sim 1.5$ in the optical window, unlike the more luminous ULIRGs such as Arp 220 which are too faint in the UV. Due to the strong UV emission of VV 114W it would be detected even at $z \sim 3$ with color, luminosity and size closely resembling those of Lyman-break galaxies. Yet, the eastern component, which as we have seen is the most energetically active, would be invisible since its UV emission is completely absorbed and re-emitted at longer wavelengths due to its high dust content (see Figs. 3 and 4 of Goldader et al. 2002). Obviously this will provide a highly distorted and unrealistic view of the system since we will be clearly unaware of the component which is responsible for the bulk of its bolometric luminosity. This would be similar to what has been observed by Ivison et al. (2001) in the SCUBA source SMMJ14011+0252, which is located at $z=2.56$ and harbors 
faint unobscured companions within an otherwise large and opaque star-forming system.

What evidence do we have though that VV 114 is representative of the high redshift galaxies? Firstly we do know that mergers and interactions were more frequent in the early Universe (Le Fèvre et al. 2000). Secondly the sources detected at $15 \mu \mathrm{m}$ in ISOCAM deep MIR surveys (Aussel et al. 1999; Elbaz et al. 1999) are mostly LIRGs rather than ULIRGs (Elbaz et al. 2002). Finally, similarly to what is observed in VV 114 , in most of the luminous infrared mergers at low- $z$ the MIR flux - and possibly the FIR as well - is found to originate mostly from one component of the merging system (Dinh et al. 2001; Charmandaris et al. 2002a,b). The above observations suggest that indeed galaxies such as VV 114 could be very relevant in probing galaxy evolution at high redshift.

\section{Conclusions}

Using our ISOCAM MIR observations of the luminous infrared merger VV 114, we conclude that:

1) Nearly $65 \%$ of the MIR emission detected in VV 114 is associated with its eastern component VV 114E. Even though most of the flux from VV 114E seems to originate from the central $1 \mathrm{kpc}$ region, we also observe a diffuse and extended component associated with the galactic disk which is not detected in the ground-based MIR images of the system. This is in stark contrast with other LIRGs/ULIRGs which typically show a compact MIR emission. The global 5-16 $\mu \mathrm{m}$ SED of the eastern component suggests that most of its mid-infrared luminosity is powered by vigorous starburst activity.

2) Almost $100 \%$ of the IRAS $12 \mu \mathrm{m}$ flux of the merger has been retrieved by ISOCAM and can be found within an area of $6 \mathrm{kpc}$ in diameter.

3) The MIR emission from the western component VV $114 \mathrm{~W}$ is more diffuse and does not peak at its nucleus. Rather, it displays a local maximum near knot 2, a bright HII region located in the overlap area of VV 114E and VV 114W.

4) The spectrum of VV 114E displays an elevated continuum at $5 \mu \mathrm{m}$ which is typically observed in MIR spectra of active nuclei as it is thought to originate from very hot dust ( $\sim 1000-1500 \mathrm{~K})$ heated by the intense radiation field encountered in the vicinity of AGNs. It could therefore reveal the presence of an AGN which, inconspicuous even at optical and radio wavelengths, contributes up to $40 \%$ of the MIR emission from the nucleus of VV 114E.

5) At kpc scales, we note a generally good agreement between the spatial distributions of the hot dust as seen in the MIR, the cold dust observed at submillimeter wavelengths as well as the molecular gas traced with the CO line. The displacement among their peaks of emission may result from temperature gradients of the dust in the overlap region of the two galaxies.

6) A LIRG similar to VV 114 placed at high- $z$ would be easily detected in optical deep surveys. Yet, the derived properties, and especially its estimated extinction, would be those of VV $114 \mathrm{~W}$ since the eastern galaxy, which dominates the FIR/bolometric luminosity is obscured in the UV and visible. Consequently, any correlation between the rest-frame UV slope of its continuum and its bolometric luminosity would be inaccurate as they would trace different components of the system.

Acknowledgements. We have greatly appreciated the assistance of R. Gastaud (Saclay) for his help on the reduction and the analysis of the ISOCAM data. We would like to thank D. Frayer (Caltech) for providing us with his SCUBA maps and N. Scoville (Caltech) for making the reduced HST/NICMOS images of VV 114 publicly available. ELF wishes to express extensive thanks to P.-A. Duc (Saclay) for fruitful discussions on numerous aspects related to this work and his careful reading of the manuscript. VC would like to acknowledge B.T. Soifer, G. Neugebauer and J. Mazzarella (Caltech) for their advice on the comparison and interpretation of ISO data with previous work as well as the support of JPL contract 960803. IFM acknowledges partial support from CONICET, Argentina. Finally, we warmly thank our referee, R. Maiolino, for carefully reviewing the paper and particularly drawing our attention on the origin of the bolometric luminosity of VV 114. Guest User, Canadian Astronomy Data Center, which is operated by the Dominion Astrophysical Observatory for the National Resarch Council of Canada's Herzberg Institute of Astrophysics.

\section{Appendix A: What is the IRAS $12 \mu \mathrm{m}$ flux of VV114?}

Since the analysis of our data revealed an extended MIR emission surrounding VV 114 we were very interested in comparing our measurements with the IRAS $12 \mu \mathrm{m}$ flux of the galaxy. Our goal was to accurately calculate what fraction of the IRAS flux was also detected by ISOCAM. The two most commonly quoted values for the $12 \mu \mathrm{m}$ IRAS flux are $0.98 \pm 0.041 \mathrm{Jy}$, from the BGS sample presented by Soifer et al. (1989), and the more recent $0.6782 \pm 10 \%$ Jy, based on the Faint Source Catalogue of Moshir et al. (1990). Recently though, Soifer et al. (2001) using the SCANPI utility provided by IPAC re-measured the $12 \mu \mathrm{m}$ IRAS flux of the galaxy over an aperture of 2 arcmin in diameter and found that it was $1.1 \mathrm{Jy}$.

Since the IRAS Si:As detectors used for the $12 \mu \mathrm{m}$ survey were $0.75 \times 4.5$ arcmin in size, we felt that it was quite likely that the measured emission from VV 114 could be contaminated by another companion galaxy. Indeed, observing the field around VV 114 we noticed that there were several sources in its proximity with one of them, IC 1622, being just 3.1 arcmin to the southwest, and having a recession velocity of $6343 \mathrm{~km} \mathrm{~s}^{-1}$ just $300 \mathrm{~km} \mathrm{~s}^{-1}$ higher than VV 114 (see Fig. 107 of Hibbard et al. 2002). IC 1622 has integrated B and V magnitudes which are just 1 mag fainter than VV 114 and since its $1.4 \mathrm{GHz}$ radio continuum flux is $7.4 \mathrm{mJy}$ (Condon et al. 1998) it should clearly form stars and emit in the MIR. The possible MIR/FIR contribution of IC 1622 was also noted by Condon et al. (1990) due to the observed offset of the IRAS centroid from the radio position of VV 114.

We proceeded by reexamine in detail the five individual IRAS scans passing near VV 114. All scans were running from the southeast to northwest direction. We noticed that the profiles of the spectra for 4 of the scans display a "bump" approximately 2.5 arcmin from the nominal position of VV 114 . The only scan which does not display this feature is scan \#3 and this is the nearest scan to the galaxy, reaching to a minimum angular distance of less than 0.5 arcmin. Integrating the line 
profile for only this scan we find that the zero crossing flux value is $0.63 \mathrm{Jy}$. The above evidence lead us to believe that the "real" $12 \mu \mathrm{m}$ flux of the galaxy, as seen by IRAS, is better represented by the point source profile fit to it as measured by the Faint Source Catalogue and hence ISOCAM has indeed detected, within our photometric uncertainties, nearly $100 \%$ of the IRAS flux.

\section{References}

Arp, H. 1966, ApJS, 14, 1

Aussel, H., Cesarsky, C., Elbaz, D., \& Starck, J. L. 1999, A\&A, 342, 313

Boselli, A., Lequeux, J., Contursi, A., et al. 1997, A\&A, 324, L13

Boulanger, F., Reach, W. T., Abergel, A., et al. 1996, A\&A, 315, 325

Cesarsky, C., Abergel, A., Agnese, P., et al. 1996, A\&A, 315, L32

Charmandaris, V., Stacey, G. J., \& Gull, G. 2002a, ApJ, 571, 282

Charmandaris, V., Laurent, O., Le Floc'h, E., et al. 2002b, A\&A, 391, 429

Combes, F. 2001, Fueling the AGN. In Lectures on the StarburstAGN Connection, INAOE, ed. D. Kunth, \& I. Aretxaga [astro-ph/0010570]

Condon, J. J., Cotton, W. D., Greisen, E. W., et al. 1998, AJ, 115, 1693

Condon, J. J., Helou, G., Sanders, D. B., \& Soifer, B. T. 1990, ApJS, 73,359

Condon, J. J., Huang, Z.-P., Yin, Q. F., \& Thuan, T. X. 1991, ApJ, 378, 65

Coulais, A., \& Abergel, A. 2000, A\&AS, 141, 533

Dale, D. A., Helou, G., Contursi, A., Silbermann, N. A., \& Kolhatkar, S. 2001, ApJ, 549, 215

Désert, F.-X., Boulanger, G., \& Puget, J.-L. 1990, A\&A, 237, 215

Dinh, V. T., Lo, K. Y., Kim, D. C., Gao, Y., \& Gruendl, R. A. 2001, ApJ, 556, 141

Doyon, R., Nadeau, D., Joseph, R. D., et al. 1995, ApJ, 450, 111

Draine, B. T. 1989, in Proc. 22nd ESLAB Symp. on IR Spectroscopy in Astronomy, ed. B. H. Kaldeich, ESA SP-290, 93

Dudley, C. C., \& Wynn-Williams, C. G. 1997, ApJ, 488, 720

Elbaz, D., Cesarsky, C., Fadda, D., et al. 1999, A\&A, 351, L37

Elbaz, D., Cesarsky, C., Chanial, P., et al. 2002, A\&A, 384, 848

Frayer, D. T., Ivison, R. J., Smail, I., Yun, M. S., \& Armus, L. 1999, AJ, 118, 139

Gallais, P., Laurent, O., Charmandaris, V., et al. 1999, in The Universe as seen by ISO, ESA SP-427, p. 880

Gao, Y., \& Solomon, P. M. 1999, ApJ, 512, L99

Genzel, R., Lutz, D., Sturm, E., et al. 1998, ApJ, 498, 579

Goldader, J. D., Meurer, G., Heckman, T. M., et al. 2002, ApJ, 568, 651

Helou, G., Lu, N., Werner, M., Malhotra, S., \& Silbermann, N. 2000, ApJ, 532, L21

Hibbard, J. E., Rupen, M. P., \& van Gorkom, J. H. 2002, in Gas and Galaxy Evolution, ed. J. E. Hibbard, M. P. Rupen, \& J. H. van Gorkom, ASP Conf. Ser., 240, 659

Hughes, D. H., Serjeant, S., Dunlop, J., et al. 1998, Nature, 394, 241

Imanishi, M., \& Dudley, C. C. 2000, ApJ, 545, 701

Imanishi, M. 2002, ApJ, 569, 44

Ivison, R. J., Smail, I., Frayer, D., Kneib, J.-P., \& Blain, A. 2001, ApJ, 561, L45
Kessler, M. F., Steinz, J. A., Anderegg, M. E., et al. 1996, A\&A, 315, L27

Knop, R. A., Soifer, B. T., Graham, J. R., et al. 1994, AJ, 107, 920

Krolik, J. 1999, in Active Galactic Nuclei, Princeton Series in Astrophysics (Princeton University Press)

Laurent, O., Mirabel, I. F., Charmandaris, V., et al. 2000, A\&A, 359, 887

Le Floc'h, E., Mirabel, I. F., Laurent, O., et al. 2001, A\&A, 367, 487

Le Fèvre, O., Abraham, R., Lilly, S. J., et al. 2000, MNRAS, 311, 565

Léger, A., \& Puget, J.-L. 1984, A\&A, 137, L5

Li, A., \& Draine, B. T. 2001, ApJ, 554, 778

Lutz, D., Spoon, H. W. W., Rigopoulou, D., Moorwood, A. F. M., \& Genzel, R. 1998, A\&A, 505, L103

Lutz, D. 1999, in The Universe as seen by ISO, ESA SP-427, p. 623

Mathis, J. S. 1990, ARA\&A, 28, 37

McMahon, R. G., Priddey, R. S., Omont, A., Snellen, I., \& Withington, S. 1999, MNRAS, 309, 1

Meusinger, H., Stecklum, B., Theis, C., \& Brunzendorf, J. 2001, A\&A, 379, 845

Mihos, J. C., \& Hernquist, L. 1996, ApJ, 464, 641

Mirabel, I. F., Vigroux, L., Charmandaris, V., et al. 1998, A\&A, 333, L1

Mirabel, I. F., Laurent, O., Sanders, D. B., et al. 1999, A\&A, 341, 667

Moshir, M., Kopar, G., Conrow, T., et al. 1990, IRAS Faint Source Catalogue, ver. 2.0.

Murphy, T. W, Soifer, B. T., Matthews, K., \& Armus, L. 2001, ApJ, 559,201

Okumura, K. 1999, in ISO beyond point sources: Studies of extended infrared emission, ESA SP-455, 47

Ressler, M. E., Werner, M. W., van, Cleve, J., \& Choa, H. 1994, Exp. Astron., 3, 277

Rieke, G. H., \& Low, F. J. 1972, ApJ, 176, L95

Rigopoulou, D., Spoon, H. W. W., Genzel, R., et al. 1999, AJ, 118, 2625

Roussel, H., Sauvage, M., Vigroux, L., \& Bosma, A. 2001, A\&A, 372, 427

Sanders, D. B., \& Mirabel, I. F. 1996, ARA\&A, 34, 749

Sanders, D. B., Soifer, B. T., \& Helias, J. H. 1988, ApJ, 325, 74

Scoville, N. Z., Evans, A. S., Thompson, R., et al. 2000, AJ, 119, 991

Soifer, B. T., Sanders, D. B., Madore, B. F., Neugebauer, G., \& Danielson, G. E. 1987, ApJ, 320, 238

Soifer, B. T., Boehmer, L., Neugebauer, G., \& Sanders, D. B. 1989, AJ, 98, 766

Soifer, B. T., Neugebauer, G., Matthews, K., et al. 2000, AJ, 119, 509

Soifer, B. T., Neugebauer, G., Matthews, K., et al. 2001, AJ, 122, 1213

Solomon, P. M., Downes, D., Radford, S. J. E., \& Barrett, J. W. 1997, ApJ, 478, 144

Starck, J. L., Abergel, A., Aussel, H., et al. 1999, A\&AS, 134, 135

Sturm, E., Lutz, D., Tran, D., et al. 2000, A\&A, 358, 481

Thornley, M. D., Förster Schreiber, N. M., Lutz, D., et al. 2000, ApJ, 539,641

Williams, R. E., Brett, B., Dickinson, M., et al. 1996, AJ, 112, 1335

Wilson, C. D., Scoville, N. Z., Madden, S. C., \& Charmandaris, V. 2000, ApJ, 542, 120

Xu, C., Gao, Y., \& Mazzarella, J., et al. 2000, ApJ, 541, 644

Yun, M. S., Scoville, N. Z., \& Knop, R. A. 1994, ApJ, 430, L109

Yun, M. S., \& Scoville, N. Z. 1995, ApJ, 451, L45 